\title{
Thermal Properties of the Heterointerface between Graphene-Like Molybdenum Disulfide and Copper
}

\author{
Jifen Wang1, Huaqing Xie ${ }^{1}$, Zhixiong Guo \\ ${ }^{1}$ School of Science, College of Art and Science, Shanghai Polytechnic University \\ No.2360 Jinhai Rd., Shanghai 201209, China \\ wangjifen@sspu.edu.cn; hqxie@sspu.edu.cn \\ ${ }^{2}$ Department of Mechanical and Aerospace Engineering, Rutgers, The State University of New Jersey \\ Piscataway, NJ 08854, USA \\ guo@jove.rutgers.edu
}

\section{Extended Abstract}

Two-dimensional materials with thin layered structures offer a remarkably rich materials platform to study the atomic level properties[1]. $\mathrm{MoS}_{2}$, as a member of the 2D material family, had received tremendous attention for their striking properties. $\mathrm{MoS}_{2}$ presents semiconducting electronic character with a direct band-gap of $1.8 \mathrm{eV}$ which has been experimentally produced in free-standing and single-layer form[1,2]. The thermal properties of 2D materials and their heterostructures are very important for efficient heat dissipation in nano-electronic devices based on 2D materials[2,3]. Based on available studies, it is evident that the incompatibility of the two different materials normally poses a strong resistance to the heat transport at the interface and is hence a major obstacle for effective heat dissipation $[4,5]$.

In this work, we built graphene like $\mathrm{MoS}_{2}$ interface structures and optimize them by first-principles for the purpose of studying how interaction and bonding of the interface atoms. The electronic structures are obtained based on first-principles density functional theory calculations. We discussed how the structure of the graphene like $\mathrm{MoS}_{2}$ interface and analyze the relationship between these coupling effects and the heat conductance. It will reveal how the bond,defects, doping, layers, and symmetry affect the heat transport of the graphene like $\mathrm{MoS}_{2}$ van Edward interface.These studies were designed to clarify the microscopic mechanism of the graphene like $\mathrm{MoS}_{2}$ Van der Waals interface heat transport. They will provide necessary scientific basis in giving full play to the superior performance of graphene like $\mathrm{MoS}_{2}$ and two-dimensional transition metal dichalcogenides in the micro-nano electronics applications for thermal design and thermal management.

\section{Acknowledgment}

This work was supported by the National Science Foundation of China (No. 51776116).

\section{References}

[1] E. D. Grayfer, M. N. Kozlova, V. E. Fedorov, "Colloidal 2D nanosheets of MoS2 and other transition metal dichalcogenides through liquid-phase exfoliation," Adv Colloid Interfac, vol. 245, pp. 40-61, 2017.

[2] X. Y. Li, S. H. Zhang, Q. Wang, "Topological insulating states in 2D transition metal dichalcogenides induced by defects and strain," Nanoscale, vol. 9, pp. 562-569, 2017.

[3] A. Lipovskii, V. Zhurikhina, D. Tagantsev, "2D-structuring of glasses via thermal poling A short review," International Journal of Applied Glass Science, vol. 9, pp. 24-28, 2018.

[4] S. Manzeli, D. Ovchinnikov, D. Pasquier, O.V. Yazyev, A. Kis, "2D transition metal dichalcogenides," Nat Rev Mater, vol. 2, p. 17033, 2017.

[5] P. Liu, B. Xiang, "2D hetero-structures based on transition metal dichalcogenides: fabrication," Sci Bull, vol. 62, pp. 1148-1161. 2017. 